\title{
Context-Specific Grasp Movement Representation in Macaque Ventral Premotor Cortex
}

\author{
Marie-Christine Fluet, ${ }^{1 \star}$ Markus A. Baumann, ${ }^{1 \star}$ and Hansjörg Scherberger ${ }^{1,2,3}$ \\ ${ }^{1}$ Institute of Neuroinformatics, University of Zürich and ETH Zürich, CH-8057 Zürich, Switzerland, and ${ }^{2}$ Deutsches Primatenzentrum GmbH and \\ ${ }^{3}$ Department of Biology, University of Göttingen, D-37077 Göttingen, Germany
}

\begin{abstract}
Hand grasping requires the transformation of sensory signals to hand movements. Neurons in area F5 (ventral premotor cortex) represent specific grasp movements (e.g., precision grip) as well as object features like orientation, and are involved in movement preparation and execution. Here, we examined how F5 neurons represent context-dependent grasping actions in macaques. We used a delayed grasping task in which animals grasped a handle either with a power or a precision grip depending on context information. Additionally, object orientation was varied to investigate how visual object features are integrated with context information. In 420 neurons from two animals, object orientation and grip type were equally encoded during the instruction epoch ( $27 \%$ and $26 \%$ of all cells, respectively). While orientation representation dropped during movement execution, grip type representation increased (20\% vs $43 \%)$. According to tuning onset and offset, we classified neurons as sensory, sensorimotor, or motor. Grip type tuning was predominantly sensorimotor $(28 \%)$ or motor $(25 \%)$, whereas orientation-tuned cells were mainly sensory $(11 \%)$ or sensorimotor $(15 \%)$ and often also represented grip type (86\%). Conversely, only $44 \%$ of grip-type tuned cells were also orientation-tuned. Furthermore, we found marked differences in the incidence of preferred conditions (power vs precision grips and middle vs extreme orientations) and in the anatomical distribution of the various cell classes. These results reveal important differences in how grip type and object orientation is processed in F5 and suggest that anatomically and functionally separable cell classes collaborate to generate hand grasping commands.
\end{abstract}

\section{Introduction}

Hand grasping is a goal-directed behavior that is largely driven by object characteristics, like shape, orientation, and size. It therefore requires the transformation of sensory information into appropriate hand motor commands. The ventral premotor cortex $(\mathrm{PMv})$ in the frontal cortex, specifically area F5, participates in such visuomotor transformations for grasping (Gentilucci et al., 1983; Murata et al., 1997; Fogassi et al., 2001; Cerri et al., 2003; Umilta et al., 2007). Anatomically, area F5 is strongly and reciprocally connected to the parietal cortex, in particular to the anterior intraparietal area (AIP) (Matelli et al., 1986; Luppino et al., 1999; Tanné-Gariépy et al., 2002; Borra et al., 2008), which also contains hand grasping signals (Taira et al., 1990; Sakata et al., 1995; Murata et al., 2000; Baumann et al., 2009). At the motor output side, F5 has direct projections to the hand area of primary motor cortex (Matsumura and Kubota, 1979; Muakkassa and Strick, 1979; Matelli et al., 1986; Dum and Strick, 2005) and to the spinal cord (Dum and Strick, 1991; He et al., 1993; Borra et al.,

Received June 23, 2010; revised Sept. 9, 2010; accepted Sept. 14, 2010.

This work was supported by the Swiss National Science Foundation (Grants 108323/1 and 120652/1), the Swiss National Center for Competence in Research "Neural Plasticity and Repair," the European Commission (International Reintegration Grant 13072 “NeuroGrasp"), the University of Zurich (Grant FK2004), the Zurich Center for Integrative Human Physiology (fellowship to M.C.F.), and the Swiss Academy of Medical Sciences (fellowship to M.A.B.). We thank B. Disler and G. Stichel for animal care and the Institute of Neuroinformatics for administrative assistance. ${ }^{*}$ M.C.F. and M.A.B. contributed equally to this work.

Correspondence should be addressed to Hansjörg Scherberger, Deutsches Primatenzentrum GmbH, Kellnerweg 4, D-37077 Göttingen, Germany. E-mail: hscherberger@dpz.eu.

DOI:10.1523/JNEUROSCI.3343-10.2010

Copyright $\odot 2010$ the authors $\quad$ 0270-6474/10/3015175-10\$15.00/0
2010). Furthermore, it is causally related to grasping execution (Fogassi et al., 2001).

Functionally, neurons in F5 are selectively active for specific grip types, like precision, whole hand, or power grips (Gentilucci et al., 1988; Murata et al., 1997; Raos et al., 2006; Stark et al., 2007). One type of cells, motor neurons, respond only during grasping execution, whereas others, so-called visuomotor neurons, also respond during visual presentation of specific objects (Murata et al., 1997). In addition, F5 activity is modulated by the object orientation (Kakei et al., 2001; Raos et al., 2006).

In these previous investigations, the shape of the object always determined the grip type to be applied. However, objects can often be grasped with various grips depending on behavioral context. Context-specific, or rule-based, information has been demonstrated in frontal cortex (White and Wise, 1999; Hoshi et al., 2000; Wallis et al., 2001; Amemori and Sawaguchi, 2006) and in parietal cortex (Gottlieb and Goldberg, 1999; Kalaska et al., 2003; Gail and Andersen, 2006; Scherberger and Andersen, 2007). Recently, we demonstrated such context-specific grasp movement signals in AIP (Baumann et al., 2009). Since AIP and F5 are intimately connected, we hypothesize that F5 also contains contextspecific information that might contribute, in addition to sensory information, to grasp movement preparation.

To test this hypothesis, we recorded single-unit activity in F5 while macaque monkeys were instructed, by an external context cue, to grasp a handle with one of two grip types (power or precision grip). In addition, object orientation was varied to investigate how visual object features are integrated with context information. We found context-dependent grasp planning activ- 
ity in F5 that appeared after cue presentation and motor grasp activity that became active at movement execution. We classified F5 neurons as sensory, sensorimotor, and motor for grip type and object orientation according to the extent of their respective tuning in the task, and found functional and anatomical differences between these groups.

\section{Materials and Methods}

Basic procedures. Single-unit activity was recorded from area F5 in two awake female rhesus macaque monkeys (animals L and J). All procedures and animal care were in accordance with the guidelines set by the Veterinary Office of the Canton of Zurich and the guidelines for the care and use of mammals in neuroscience and behavioral research (National Research Council, 2003).

Methods have been extensively described previously (Baumann et al., 2009). In short, monkeys were seated in a primate chair and trained to perform a grasping task with their left hand. They faced a handle placed at the level of the chest and at a distance of $\sim 30 \mathrm{~cm}$. The handle could be grasped either with a power grip (opposition of fingers and palm) or a precision grip (opposition of index and thumb pads). Inside the handle, two touch sensors were placed in small, clearly visible recessions to detect the contact of the animal's thumb and index finger during precision grips (Fig. $1 \mathrm{~A}$, red dot; only one sensor visible). Power grips were detected by an infrared light barrier inside the opening of the handle (Fig. $1 A$, red line). Grip type was instructed by two colored LEDs that were projected on the center of the handle by a half mirror positioned between the animal's eyes and the target. The handle was rotatable and was presented in five different orientations (upright and $25^{\circ}$ and $50^{\circ}$ to the left and right) and two spotlights could illuminate it from the left and right side in an otherwise dark experimental room. Two capacitive touch sensors (model EC3016NPAPL; Carlo Gavazzi) were placed at the level of the animals' waist as handrest buttons. Animals had to fixate a red LED that was projected on the center of the handle. Eye movements were measured using an optical eye tracker (ISCAN) and custom-written software implemented in LabView Realtime (National Instruments) was used to control the behavioral task.

Behavioral paradigm. Monkeys were first trained in a delayed grasping task in which they were required to grasp a handle in five possible orientations (upright and $25^{\circ}$ and $50^{\circ}$ to the right or left) with either a power grip or a precision grip. This led to 10 grasp conditions that were presented in a pseudorandom order. To initiate a trial, monkeys sat in darkness and placed each hand on a handrest button. The handle was then positioned in one of the five orientations and subsequently a red fixation LED switched on. From then on, the animal was required to fixate while keeping both hands at the handrest buttons (fixation period: duration, $700-1100 \mathrm{~ms}$; mean, $900 \mathrm{~ms}$ ). In the following cue period (duration, 600 $\mathrm{ms}$ ), the object was illuminated to reveal its orientation. The color of an additional LED presented next to the fixation LED indicated which grip type to perform, either a power grip (green light) or a precision grip (white light). The spotlights and the instructing LED were then switched off while the fixation light remained on for a variable period (duration, $700-1100 \mathrm{~ms}$; mean, $900 \mathrm{~ms}$ ) during which the monkey had to remember the grasping instructions (planning period). The red fixation LED was then dimmed, which instructed the animal to reach and grasp the object in the dark. The movement period started with the go-signal and ended when the sensors on the handle were activated. Each correct trial was rewarded with a fixed amount of juice, which was delivered imme-

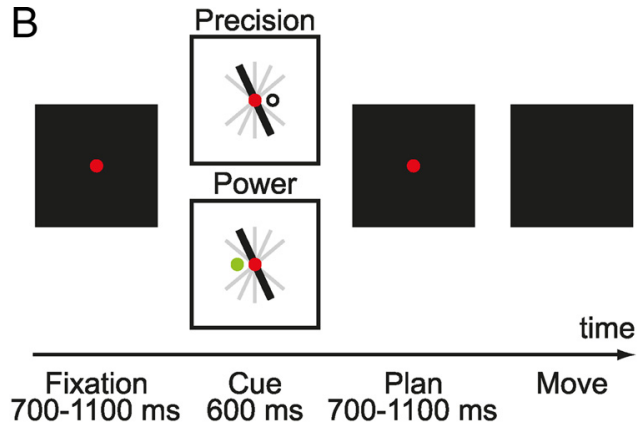

D

$\mathrm{E}$
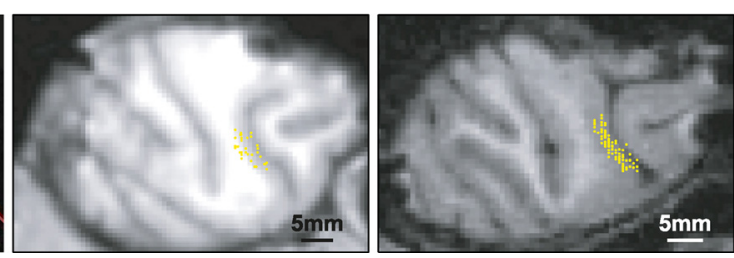

Figure 1. Task paradigm and recording sites. $\boldsymbol{A}$, Picture of the handle. Two sensors in grooves (red circle, only one sensor visible) visibeand colored LED indicates the grip type (power or precision). The lights are projected onto the middle of the handle through hemisphere of monkey $L$ in the plane of the recording chamber. Superimposed on the image are the recording sites in area $F 5$ (yellow dots). $\boldsymbol{E}$, Same as $\boldsymbol{D}$ for monkey J.

diately after the grasp target was correctly acquired. The trial sequence is presented in Figure $1 B$.

Surgical procedures and imaging. When the animal was fully trained, an MRI scan was performed to locate anatomical landmarks for the implantation of a recording chamber. For this, the animal was sedated (ketamine and xylazine, 10 and $0.5 \mathrm{mg} / \mathrm{kg}$, i.m., respectively), placed in the scanner (GE Healthcare 1.5T) in a prone position, and T1-weighted volumetric images were obtained of the brain and skull (Baumann et al., 2009). The stereotaxic location of the arcuate sulcus was then obtained to guide the placement of the recording chamber.

Next, a surgery was performed to implant a head post (titanium cylinder, $18 \mathrm{~mm}$ diameter) and an oval-shaped recording chamber (material PEEK; outer dimensions, $40 \times 25 \mathrm{~mm}$ ) stereotaxically over the right hemisphere on top of F5; both were secured to the skull with titanium bone screws. Initially, no skull trephination was performed. After recovery from surgery, a second MRI scan was obtained with the chamber filled with contrast medium (Gadolinium solution diluted in saline, 1:2000). This allowed mapping of the brain inside of the recording chamber. Finally, a second surgery was performed for the craniotomy. Recordings were performed in the posterior bank of the inferior limb of the arcuate sulcus (area F5). Figure 1, C-E, show a coronal view of monkey J and recording maps in the plane of the recording chamber for monkey $\mathrm{L}$ and J, respectively.

All surgical procedures were performed under sterile conditions and general anesthesia (induction with ketamine $10 \mathrm{mg} / \mathrm{kg}$, i.m., atropine $0.05 \mathrm{mg} / \mathrm{kg}$, s.c., followed by intubation, isofluorane $1-2 \%$, and analgesia with $0.01 \mathrm{mg} / \mathrm{kg}$ buprenorphene, s.c.). Heart and respiration rate, electrocardiogram, $\mathrm{O}_{2}$ saturation, and body temperature were continuously monitored, and systemic antibiotics and analgesics were administered for several days after each surgery. Animals were allowed to recover for at least 1 week before behavioral training or recording experiments commenced.

Neural recordings. Single-unit (spiking) activity was recorded using platinum/tungsten electrodes with quartz-glass coating (impedance, 1-2 $\mathrm{M} \Omega$ at $1 \mathrm{kHz}$ ) that were positioned by a five-channel micromanipulator (MiniMatrix; Thomas Recording) that was attached to the recording 
A

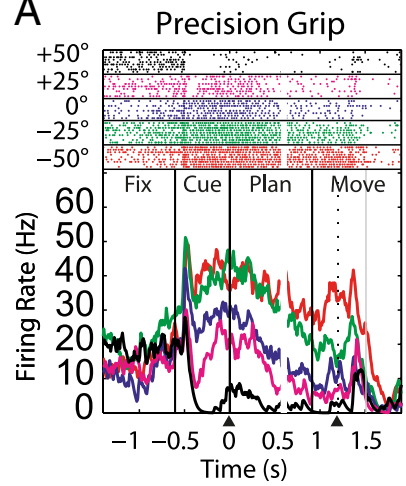

C
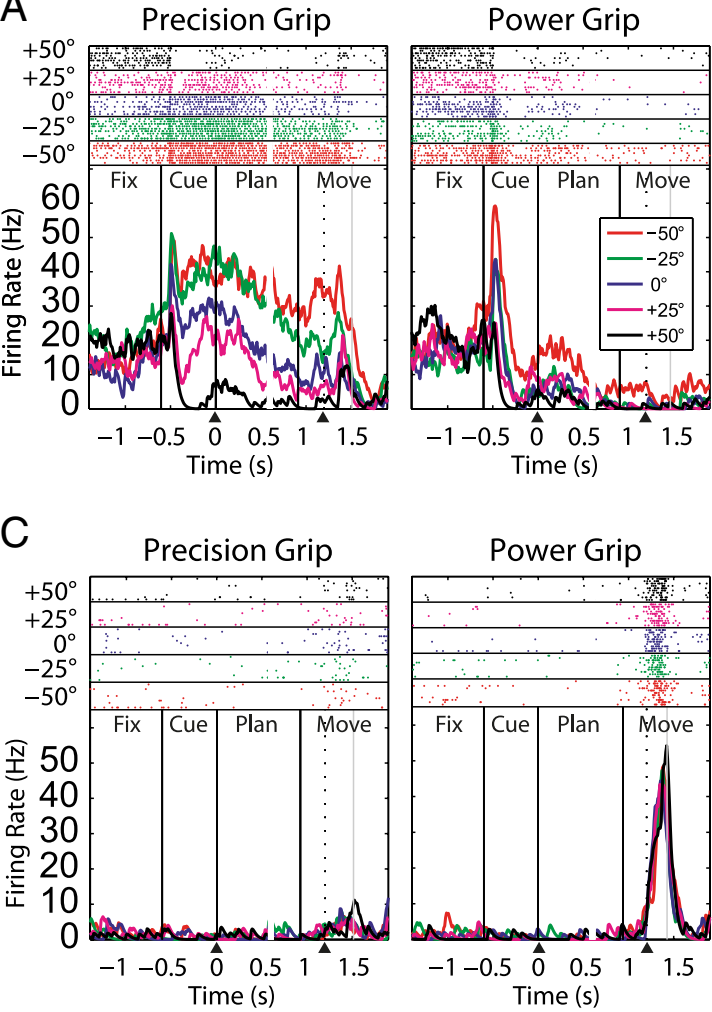

$\mathrm{B}$

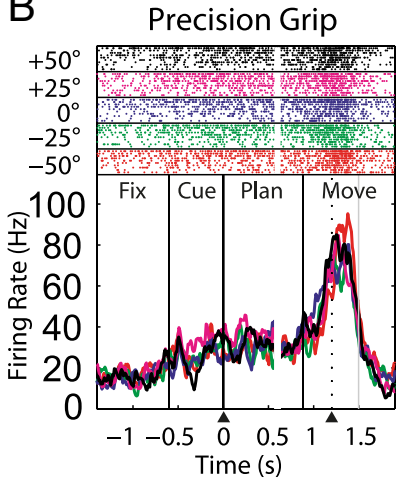

$\mathrm{D}$

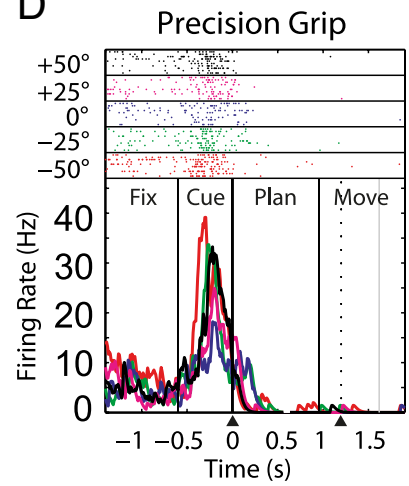

Power Grip

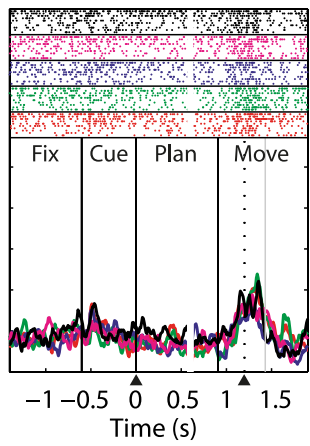

Power Grip

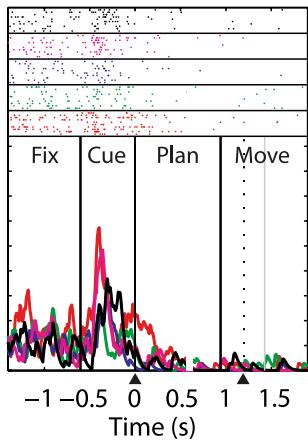

Figure 2. Firing rate histograms and raster plots of four example neurons. $\boldsymbol{A}-\boldsymbol{D}$, Left, Precision grip trials. Right, Power grip trials. The five orientations are represented by different colors. Graphs are doubly aligned to the end of the cue and the release of the handrest button (black arrowheads). Realignment occurs at $0.6 \mathrm{~s}$ and is marked by a gap in the curves and the spike rasters. Black vertical lines mark cue onset, cue offset, and the go-signal. The dotted line within the movement epoch indicates the handrest release and the gray line the (mean) time of handle contact. $\boldsymbol{A}$, Neuron modulated by grip type and orientation from the cue until completion of the task. $\boldsymbol{B}$, Neuron modulated by grip type only, starting from the cue until task completion. C, Neuron showing no task modulation during cue and planning, but clear grip-type tuning during movement execution. $\boldsymbol{D}$, Neuron modulated by grip type and orientation exclusively in the cue period.

chamber. Neural signals were amplified $(\times 400)$, digitized with 16-bit resolution at $30 \mathrm{kS} / \mathrm{s}$ using Cerebus neural signal processor (Blackrock Microsystems), and stored to disc together with the behavioral data. To coarsely monitor the tuning properties of the recorded neurons during data acquisition, spike detection was performed in real time (Cerebus hardware) and analyzed for various task conditions using Matlab (MathWorks). However, all quantitative analysis was performed offline, as described below.

Data analysis. Raw signals were high-pass filtered offline (cutoff, 500 $\mathrm{Hz}$ ) and single units were isolated using principal component analysis techniques (offline sorter; Plexon). We included in our database all single units that were stably recorded during at least seven trials per condition (minimum of 70 trials). Peristimulus-time histograms were generated using a gamma distribution as a causal kernel (parameters: shape $\alpha=1.5$; rate $\beta=30$ ) (Baumann et al., 2009). Population histograms were obtained by averaging individual peristimulus-time histograms for the entire population. All statistical tests, however, were based on exact spike counts without any smoothing.

For the generation of the population-averaged histogram, the preferred and nonpreferred grip type and orientation were determined for each cell from the mean firing rates in the time interval from cue onset to the end of the movement, which were averaged across all trials of the same grip type or object orientation. The preferred grip type was then defined as the grip with the highest mean rate and the nonpreferred grip type was the other one. The preferred orientation was defined as the orientation with the highest mean firing rate and the nonpreferred orientation was taken as the orientation at $75^{\circ}$ angular distance from the preferred one. This definition was chosen so that the nonpreferred direction was not taken exclusively from the extreme orientations $\left( \pm 50^{\circ}\right)$. For the vertical position we selected randomly between the $-50^{\circ}$ and $50^{\circ}$ position.

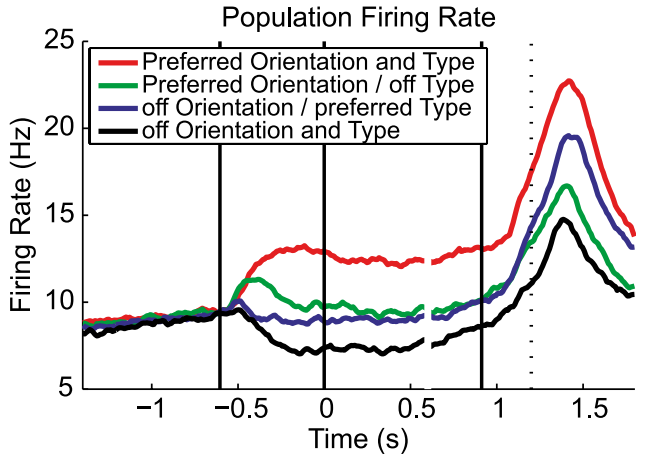

Figure 3. Population firing rate of the 420 cells during the preferred and nonpreferred conditions (grip type and orientation). Alignment and definition of time epochs as in Figure 2.

To determine the distribution of preferred conditions in the population, the same definition of preferred grip type and orientation was used, but based on the firing rate in the epoch in question or in the time interval from tuning onset to offset.

To test the statistical significance of tuning for grip type and object orientation in a particular task epoch, we first determined in each trial the mean firing rate (spike count/length of epoch) and then performed a two-way ANOVA (factors grip type and orientation, $p<0.01$ ). Only positive main effects were taken into account. In addition, we required that the neuron fired at least with five spikes/s in the preferred condition.

At the population level, we used a two-proportion $z$ test (two-tailed version) to determine whether the number of cells with significant tuning (grip type or orientation) significantly changed between two task epochs (Zar, 1999). 
Table 1. Grip-type and orientation tuning across epochs

\begin{tabular}{|c|c|c|c|c|c|c|c|}
\hline \multicolumn{4}{|c|}{ Orientation tuning } & \multicolumn{4}{|c|}{ Grip-type tuning } \\
\hline Cue & Planning & Movement & Number of cells & Cue & Planning & Movement & Number of cells \\
\hline+ & + & + & $33(8 \%)$ & + & + & + & $42(10 \%)$ \\
\hline+ & + & - & $28(7 \%)$ & + & + & - & $22(5 \%)$ \\
\hline+ & - & + & $8(2 \%)$ & + & - & + & $16(4 \%)$ \\
\hline+ & - & - & $41(10 \%)$ & + & - & - & $35(8 \%)$ \\
\hline- & + & + & $12(3 \%)$ & - & + & + & $29(7 \%)$ \\
\hline- & + & - & $29(7 \%)$ & - & + & - & $19(5 \%)$ \\
\hline- & - & + & $29(7 \%)$ & - & - & + & $93(22 \%)$ \\
\hline- & - & - & $240(57 \%)$ & - & - & - & $164(39 \%)$ \\
\hline
\end{tabular}

Number (and percentage) of cells for possible combinations of orientation tuning and grip-type tuning during the cue, planning, and movement epochs. Significant tuning is indicated by + , no tuning by - .

For this, the SE of the sampling distribution difference between two proportions was calculated as $\mathrm{SE}=\sqrt{p \times(1-p) \times\left[\left(\frac{1}{n_{1}}\right)+\left(\frac{1}{n_{2}}\right)\right]}$, with $p=\frac{n_{1} \times p_{1}+n_{2} \times p_{2}}{n_{1}+n_{2}}$ the pooled sample proportion, $n_{1}$ and $n_{2}$ the size of samples 1 and $2\left(n_{1}=n_{2}=420\right.$ cells $)$, and $p_{1}$ and $p_{2}$ the first and second sample proportion. From this, the $z$ score was calculated as $z=\frac{p_{1}-p_{2}}{\mathrm{SE}}$ and the corresponding $p$ value obtained from the (cumulative) normal distribution.

We further investigated the significance of grip type and orientation tuning in a sliding window analysis. We performed a two-way ANOVA $(p<0.01)$ in a 200 -ms-width window that was shifted by $50 \mathrm{~ms}$. In plots, the number of significant cells in each bin was reported at the center position of each window. Due to the variable length of the planning period, trials were doubly aligned for this analysis; up until $0.6 \mathrm{~s}$ after cue off, they were aligned to the cue offset, after that, they were aligned to the release of the handrest button. This approach proved to be very uncritical, as no cell showed any specific activity change around the time of realignment. Analyses based on the stepping window ANOVA (like the tuning onset/offset, see below) therefore inherited this realignment.

The time of half-maximum activity during the cue period was calculated as the time when the number of tuned cells reached the middle between the initial value at cue onset and the maximum during the cue period. For this calculation, the number of significantly tuned cells, measured at $50 \mathrm{~ms}$ steps, was linearly interpolated to reach a time resolution of $2 \mathrm{~ms}$ (Matlab command interp). Then the time delay between the start of orientation and the start of grip type activity was taken as the difference between the two half-maximum times. To assess the significance of the observed difference, we used a Monte Carlo method that calculated the above mentioned time delay at each time step 1000 times with the labels "grip type tuning" and "orientation tuning" randomly shuffled. The 95th percentile of this null-distribution was then taken as the threshold for significance.

Tuning onset for grip type or orientation was determined from the two-way ANOVA analysis and was defined as the first of at least five consecutive windows with significant tuning. Similarly, tuning offset was defined as the last of at least five such consecutive windows. Cells were classified according to their tuning onset (t_on) and offset ( $t$ _off) as sensory (t_on $<$ t_off $<0.7 \mathrm{~s}$ ), sensorimotor (t_on $<0.7 \mathrm{~s}<\mathrm{t}$ _off), or motor $\left(\mathrm{t} \_\right.$off $>\mathrm{t} \_$on $>0.7 \mathrm{~s}$ ). All times were relative to the end of the cue epoch. The combined encoding of grip type and orientation was investigated using joint distributions.

\section{Results}

\section{Tuning for grip type and orientation}

We recorded a total of 420 single units from area F5 in the right hemisphere of two monkeys (monkey L, 113 units; monkey J, 307 units) during a delayed grasping task, in which two grip types (power and precision grip) were performed with five different object orientations. Four typical neurons are presented in Figure 2. The first neuron was jointly modulated by orientation and grip type, starting shortly after cue onset and extending until move-
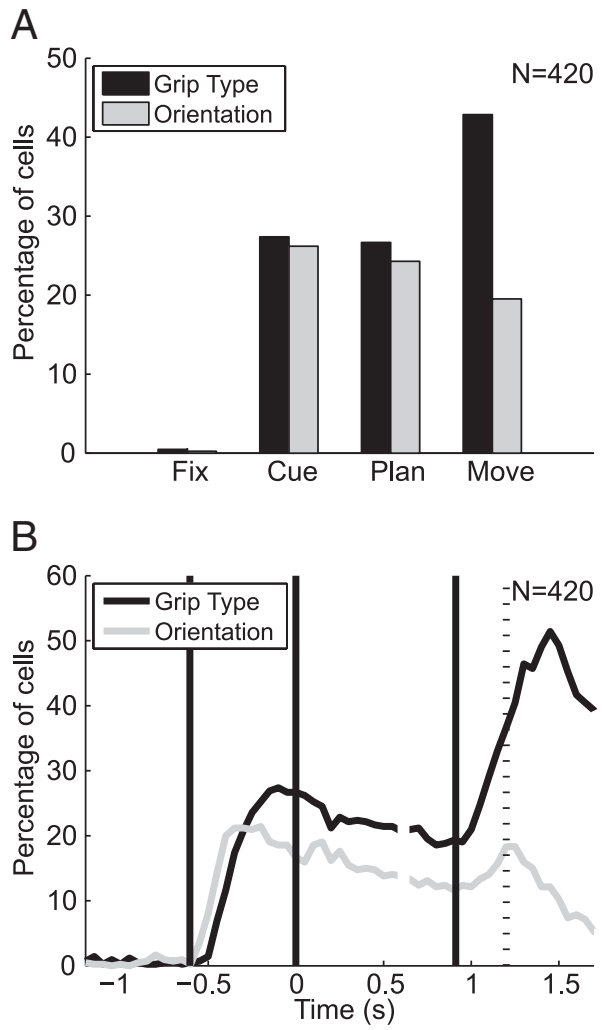

Figure 4. Grip type and orientation tuning in the population $(n=420)$. A, Percentage of cells that are tuned for grip type (black) and object orientation (gray) during each task epoch (fixation, cue, planning, and movement) (ANOVA, $p<0.01$ ). B, Percentage of tuned cells for grip type (black) and object orientation (gray) in 200 ms time windows (ANOVA, $p<0.01$; curves report data at the center of each window). Alignment and definition of time epochs as in Figure 2.

ment execution was finished. Clearly, this neuron preferred precision grips with the handle tilted to leftward orientations $\left(-25^{\circ}\right.$ and $-50^{\circ}$ ). The second neuron (Fig. $2 B$ ) showed an increased firing rate specifically for precision grip trials, independent of handle orientation. It represented only the grip type, but not the object orientation, from cue presentation until the end of the movement. The third neuron encoded the grip type with a preference for power grip, however, only during movement execution. Finally, the fourth neuron was modulated by grip type and orientation exclusively during the cue epoch. These example neurons reflect main classes of F5 neurons with sensory, sensorimotor, and motor activity.

Condition-specific modulation of the firing rate was also seen in the neural population. Figure 3 shows a population histogram of all 420 recorded cells, in which the preferred and nonpreferred 
A Cue
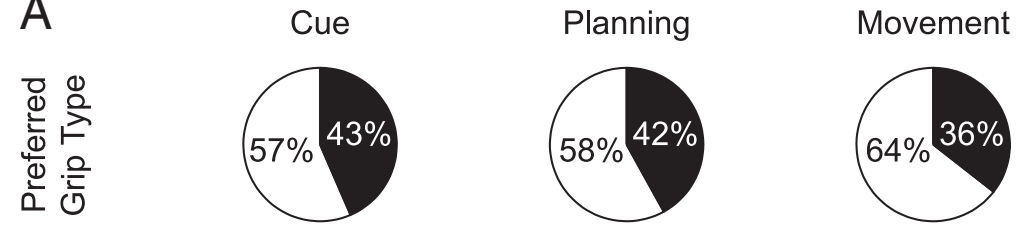

Precision Power
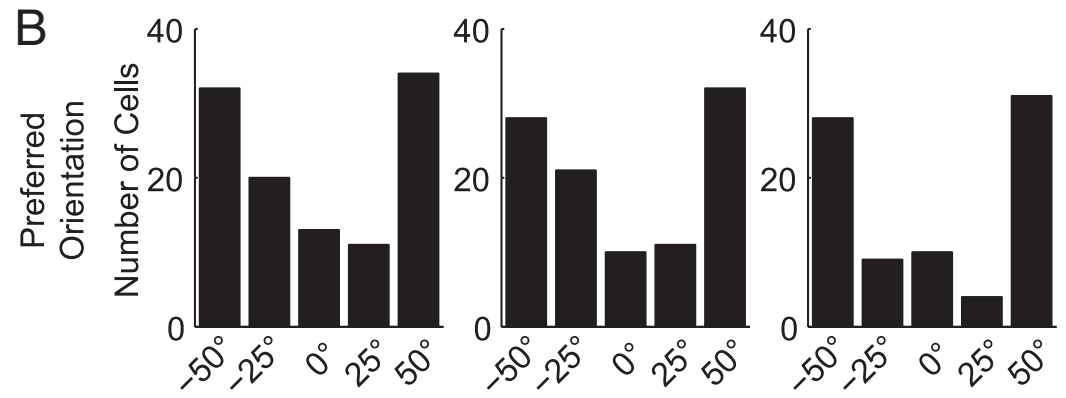

Figure 5. Preferred grip type and orientation of significantly tuned cells in each task epoch. $A$, Number of grip type-specificcells preferring precision (white) and power grip (black) during the cue, planning, and movement epoch. $\boldsymbol{B}$, Number of orientationspecific cells preferring individual object orientations $\left(-50^{\circ},-25^{\circ}, 0^{\circ}, 25^{\circ}, 50^{\circ}\right)$ during cue, planning, and movement.
A Grip type tuning consistence

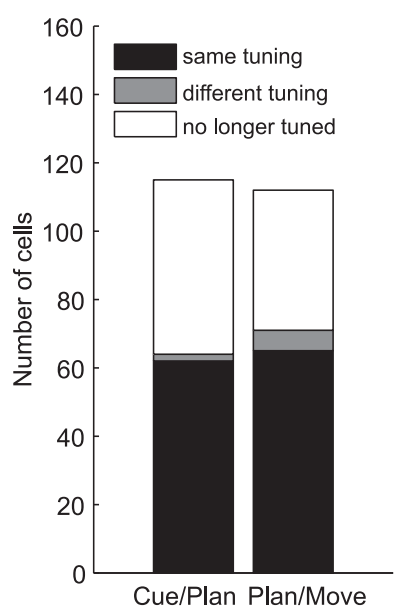

B Change of orientation tuning
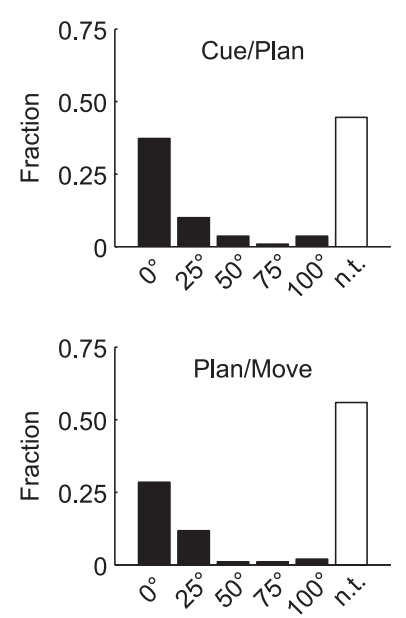

Figure 6. Tuning consistency between different task epochs. A, Grip type tuning consistency. Number of cells that kept (black), altered (gray), or lost (white) their tuning from cue to planning and from the planning to the movement epoch. $\boldsymbol{B}$, Orientation tuning consistency. Fraction of cells as a function of shift from their preferred orientation (in degrees) from cue to planning and from planning to movement. n.t., No longer tuned.

grip types and orientations were pooled separately. It demonstrates that the handle orientation and the context-cued grip type were represented in the F5 population starting from the cue epoch, long before motor execution. Results stayed the same when the activity of individual cells was normalized before pooling, confirming that the result did not rely on a few cells with particularly strong responses.

\section{Epoch analysis}

To quantify grip type and orientation tuning of individual F5 neurons in each task epoch (cue, planning, and movement), we performed a two-way ANOVA (factors grip type and object orientation, $p<0.01$ ) on the firing rate in each task epoch. Table 1 reports the frequency of the different tuning patterns in the three task epochs. Overall, $71 \%$ of the neurons were tuned for at least
Movement

one of these two parameters in at least one epoch. Specifically, $61 \%$ of the cells were tuned for grip type and $43 \%$ were tuned for object orientation.

Grip type and orientation tuning did not only show a large difference of representation ( $61 \%$ vs $43 \%$ ), but also different progression during the task (Fig. 4A). While in the cue period, the percentage of grip-type tuned neurons was the same as for orientation-tuned neurons $(27 \%$ vs $26 \%$ ), grip-type-specific neurons became more frequent during the course of the task (planning epoch, 27\%; movement, $43 \%$ ), whereas the number of orientation-specific neurons decreased (planning epoch, 24\%; movement, 20\%). A two-proportion $z$ test revealed that the number of grip-type-specific cells did not change between the cue and planning epoch $(p=0.8)$, but it increased significantly between planning and movement ( $p \ll 0.05)$. In contrast, the number of orientation-specific cells tended to decrease during the task (cue to movement, $p=0.02$ ).

Cells without significant tuning for grip type or orientation often showed a task modulation with respect to baseline activity, which was unspecific for the tested conditions (cue epoch, $49 \%$ of all nontuned cells; planning, 64\%; movement, 78\%; $t$ test, $p<$ $0.01)$. These neurons could possibly be tuned for grasp conditions other than the ones tested here. However, it is interesting to note that unspecific responses during the cue and planning epoch often consisted of a decrease below baseline activity (cue, 88 cells lower, 38 cells larger; planning, 109 lower, 61 larger), while during movement execution the majority of untuned cells increased their activity compared with baseline ( 68 lower, 97 larger). Some of these neurons might therefore constitute a timing signal and represent temporal information independent of a specific grip type.

We then investigated the representation of grip type and orientation in F5 with a finer time resolution using a two-way ANOVA in a small time window that was shifted along the time axis (window size, $200 \mathrm{~ms}$; step size, $50 \mathrm{~ms}$; $p<0.01$ ). We found that both the number of orientation and grip-type tuned cells showed a steep increase during the cue period; however, orientation tuning appeared earlier than the grip-type representation (Fig. $4 B$ ). Using the time difference between the half maxima of the two ascending curves, we quantified that orientation tuning was leading grip-type tuning by $94 \mathrm{~ms}$ in the population ( $\mathrm{p} \ll$ 0.01, Monte Carlo procedure; see Materials and Methods). Orientation information therefore appeared more rapidly in F5 than the grip-type instruction, which had to be inferred abstractly from an LED color. This can be seen also in the population histogram (Fig. 3), where the curves indicating the preferred and nonpreferred object orientation split earlier than the curves of the preferred and nonpreferred grip types. At the end of the cue period, however, the number of cells representing grip type was higher than for orientation (Fig. 4B). Both groups tended to decrease during the planning epoch. Yet, during movement execution, the number of cells representing grip type approximately doubled, whereas orientation tuning recovered only slightly. It therefore seems that grip type representation is the overall dom- 
inant feature in F5, even though orientation information appears first.

\section{Specific coding in task epochs}

To investigate the preference for specific grip types and orientations, we examined their distribution across different task epochs (cue, planning, movement). We found an overrepresentation of precision grip- versus power grip-preferring cells (Fig. 5A) with a tendency to increase from the cue (57\% precision) to the movement epoch (64\% precision). For orientation tuning, we found a predominance of extreme orientations $\left( \pm 50^{\circ}\right)$ over middle orientations $\left(-25^{\circ}, 0^{\circ}\right.$, and $\left.25^{\circ}\right)$ (Fig. $5 B$ ). Although the number of neurons coding for extreme orientations remained approximately constant during the task, neurons representing middle orientations were clearly reduced during movement execution, which resulted in a relative increase of cells preferring extreme orientations from cue $(60 \%)$ to movement execution $(72 \%)$. These changes in representation suggest functional dynamics in the coding of F5 that we examine below.

We first asked whether these shifts in the distributions of preferred grip type and orientation in F5 were due to shifts in the preference of individual neurons across the task or to different subpopulations being active in different task epochs. For this, we determined the percentage of tuned neurons that maintained or changed their specific tuning from one task epoch to the next (cue-planning, planning-movement). For grip-type tuning, very few cells changed their tuning preference; most cells either maintained their grip-type preference or lost their tuning (Fig. 6A). Similarly, the preferred orientation of individual cells was nearly constant across task epochs (Fig. 6B).

Tuning shifts occurred mainly to neighboring orientations, for which the firing rate was often not significantly different. However, a considerable number of cells lost their orientation tuning from cue to planning and planning to movement. Therefore, since most cells encoded grip type and object orientation consistently across task epochs, the observed changes in the statistics of the preferred conditions (Fig. 5) must be caused by different subpopulations of cells that are active at different times in the task.

\section{Cell classification}

Based on the sliding window analysis described above (Fig. 4B), we defined the tuning onset of an individual neuron as the first occurrence of five consecutive tuned windows. Figure 7, $A$ and $B$, shows the time windows with significant tuning for all cells in the population. For grip type (Fig. 7A) and object orientation (Fig. $7 B$ ), cells are ordered according to their tuning onset. Corresponding histograms of tuning onset (Fig. $7 C, D$ ) revealed three
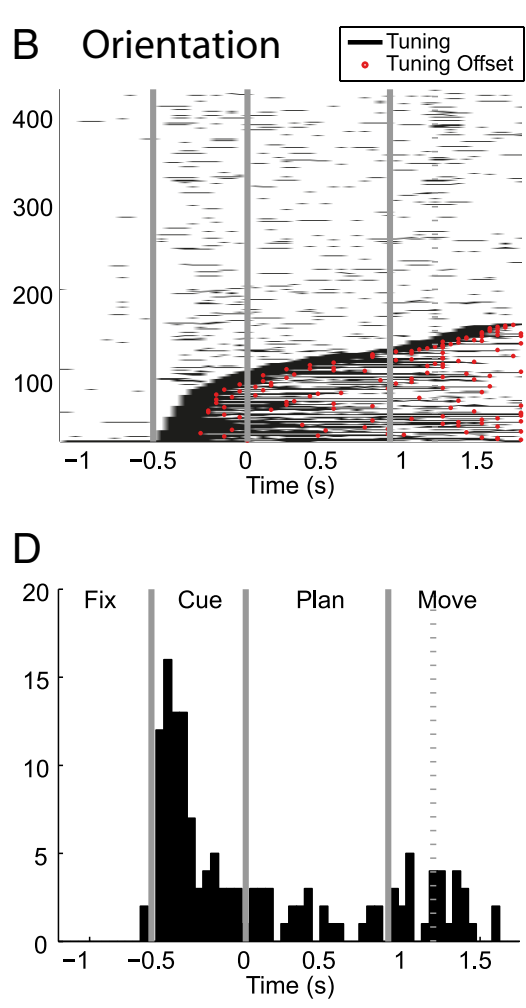

F

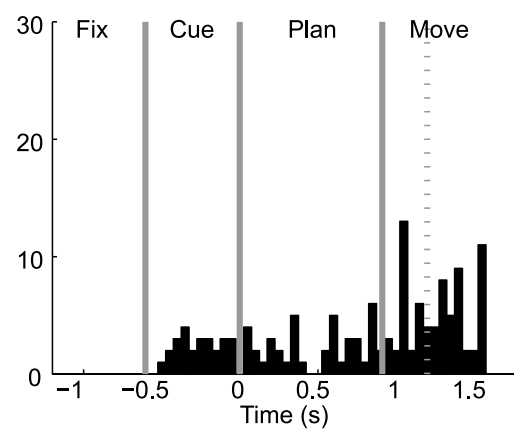

Figure 7. Time of significant tuning of grip type and orientation. $\boldsymbol{A}$, Sliding window analysis indicating the times with signifiwindow). (ells are ordered by tuning onset; red dots indicate tuning offset. $\boldsymbol{B}$, Same as $\boldsymbol{A}$, for orientation tuning. $\boldsymbol{C}-\boldsymbol{D}$, Histograms of tuning onset of grip type and orientation. $\boldsymbol{E}-\boldsymbol{F}$, Histograms of tuning offset for grip type and orientation.

important characteristics of F5 activity: (1) one group of cells showed an early tuning onset for grip type, (2) another group showed a late tuning onset for grip type, and (3) one group showed an early tuning onset for orientation. These groups are illustrated by the example neurons in Figure 2. Furthermore, tuning offset was defined as the last occurrence of five consecutive tuned windows and is indicated by a red dot in Figure 7, $\mathrm{A}$ and $B$. The corresponding tuning offset histograms (Fig. $7 E, F$ ) indicate that grip-type tuned cells remained tuned predominantly until movement execution, whereas orientation tuned cells faded off during the course of the task.

To relate tuning onset and offset of individual cells, we generated scatter plots that describe their joint distribution (Fig. 8). For grip-type tuning (Fig. $8 \mathrm{~A}$ ), the joint distribution suggested a classification of three neuronal classes: (1) sensory (S) cells with early onset and offset of tuning ( $t \_o n<t \_o f f<0.7$ s), (2) sensorimotor (SM) cells with early tuning onset and tuning persistence until movement execution (t_on $<0.7 \mathrm{~s}<\mathrm{t} \_$off), and (3) motor (M) 
A

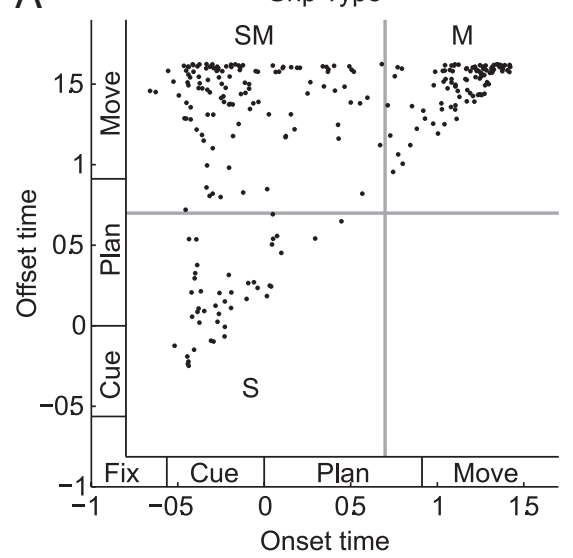

B

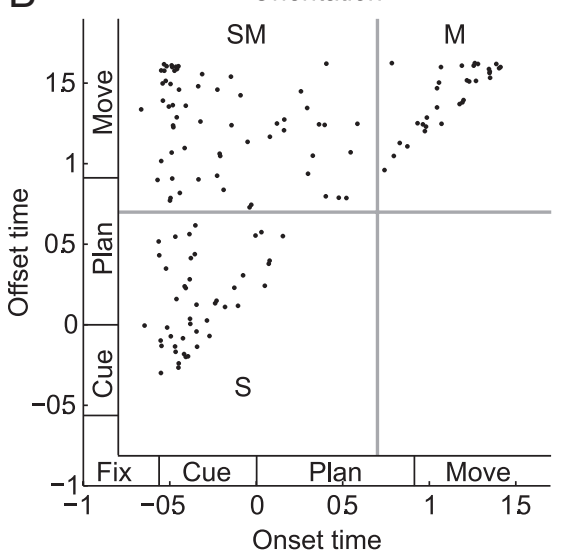

Figure 8. Scatter plots of tuning onset and offset. $A, B$, Cell classification based on the tuning onset and tuning offset for grip type $(\boldsymbol{A})$ and orientation $(\boldsymbol{B})$. The separation between the $\mathrm{S}, \mathrm{SM}$, and $\mathrm{M}$ classes is set at $0.7 \mathrm{~s}$ (gray lines).

Table 2. Joint distribution of cell classes for grip type and object orientation

\begin{tabular}{llllll}
\hline & Type & & & \\
\cline { 2 - 5 } Orientation & S & SM & M & Never & Total \\
\hline S & 10 & 20 & 7 & 8 & $45(11 \%)$ \\
SM & 10 & 39 & 8 & 4 & $61(15 \%)$ \\
M & 3 & 9 & 13 & 7 & $32(8 \%)$ \\
Never & 20 & 51 & 79 & 132 & $282(67 \%)$ \\
Total & $43(10 \%)$ & $119(28 \%)$ & $107(25 \%)$ & $151(36 \%)$ & $420(100 \%)$ \\
\hline
\end{tabular}

Joint distribution of sensory, sensorimotor, motor, and untuned (Never) cells for grip type (columns) and object orientation (rows) in the neural population. Percentages are relative to the entire population (420 cells).

cells with late tuning onset ( $\mathrm{t} \_$off $>\mathrm{t} \_$on $>0.7 \mathrm{~s}$ ). The exact threshold level (we chose $0.7 \mathrm{~s}$ after cue offset) was thereby not critical. Any value between 0.4 and $1 \mathrm{~s}$ returned very similar results, as the tuning onset and offset histograms suggest (Fig. 7CF). Using this classification scheme, we found $43 \mathrm{~S}$ cells $(10 \%)$, 119 SM cells (28\%), $107 \mathrm{M}$ cells (25\%) for grip-type tuning, and 151 untuned cells (36\%).

Likewise, the same classification scheme was applied for orientation tuning (Fig. 8 B), which gave us 45 (11\%) S cells, 61 (15\%) SM cells, 32 (8\%) M cells, and 282 (67\%) untuned cells for object orientation.

This classification expands previous schemes that distinguish only between motor and visuomotor cells, but do not recognize purely sensory cells due to the different nature of their task (Murata et al., 1997; Raos et al., 2006).

The joint distribution of grip-type and orientation classes is shown in Table 2. It demonstrates a highly significant interaction between the type and orientation classes (Pearson's $\chi^{2}, p \ll$ 0.01 ). Most orientation-tuned cells were of $S$ and SM class (106 of 138 cells), and a large majority of them were also grip-type tuned (94 of 106 cells), mainly in the S or SM class (79 of 106 cells). In contrast, many of the cells with early grip-type tuning (S and SM) were never orientation tuned (71 of 162), and if they were, they were predominantly of S or SM class for orientation (79 of 91 cells). These results are remarkably different from AIP, where a considerable number of visual neurons represent the object orientation alone, independent from grip type (Baumann et al., 2009). Moreover, in F5, an overwhelming majority of M cells for grip type was never orientation tuned (79 of 107 cells). This suggests that object orientation information is relevant in F5 primarily during grasp planning ( $\mathrm{S}$ and SM cells), but much less during movement execution ( $\mathrm{M}$ cells).

\section{Specific coding of cell classes}

The specific coding of the different cell classes showed marked differences for $\mathrm{S}$, $\mathrm{SM}$, and $\mathrm{M}$ cells (Fig. 9). For grip-type tuning (Fig. 9A), S cells were tuned for precision and power grips equally often, whereas SM and $M$ cells showed a clear overrepresentation of precision grips. An overrepresentation of precision grips has been described in motor areas and could be due to the fact that complex grip types may need more neural resources for planning and execution than simpler grips (Muir and Lemon, 1983; Lemon et al., 2004; Umilta et al., 2007). In contrast, the balanced ratio of $S$ cells suggests that these cells might employ a more abstract representation with equal resources for both grips.

A similar difference appeared for orientation tuning (Fig. 9B). S cells showed a rather balanced representation of extreme and middle orientations (53\% vs $47 \%$ ), whereas SM and M cells coded predominantly for extreme orientations ( $75 \%$ and $85 \%$, respectively). This overrepresentation of extreme orientations could reflect a motor coding along the pronation-supination dimension, whereas the more balanced representation would be compatible with a visual representation of orientation.

Together, these results suggest that SM and M cells represent motor-related signals, whereas $S$ cells may convey a more sensory or cognitive representation of grip type and object orientation. Interestingly, such a balanced representation of grip type and object orientation was also found in AIP for cells with an early tuning onset in this task (Baumann et al., 2009). This suggests that AIP cells with early tuning onset are particularly coupled with $\mathrm{S}$ cells in F5.

\section{Anatomical cell distribution}

Finally, we tested whether the cell classes have different anatomical distributions within F5. For this, we ordered all cells in the population along their dorsoventral recording position along the arcuate sulcus and distributed them into eight bins such that each bin contained the same number of cells. In each of these bins, we determined the fraction of S, SM, and M cells for grip type and orientation and found that early and late onset tuned cells had complementary distributions along the arcuate sulcus (Fig. 10). Cells with early grip type tuning ( $\mathrm{S}$ and SM cells) were concentrated mainly in two areas: the most ventral recording site (Fig. 10, top left, bins 7-8) and a dorsal site (Fig. 10, top left, bins 2-3). $S$ and SM cells with early orientation tuning showed a similar distribution with peaks in bins 2 and 8 (Fig. 10, top right). In contrast, cells with late tuning onset ( $\mathrm{M}$ cells) were concentrated at recording sites in between the early (S and SM) cells. This can be best seen for cells with late grip-type tuning (Fig. 10, bottom left, peaks in bins 4-6 and bin 1) and somewhat less clearly for $M$ cells of orientation tuning due to their smaller group size (Fig. 10, bottom right). A similar analysis in the direction normal to the sulcus orientation and in the depth direction showed no concentration effects (data not shown).

These results suggest that sensory input is not uniformly present across F5 but rather concentrated in some subregions. These subregions seem to coincide with subareas of F5 with strong connectivity to AIP. Indeed, Figure 3 in Borra et al. (2008) suggests that the anatomical projections from AIP to F5 are not 
uniformly distributed along the inferior ramus of the arcuate sulcus; although the authors did not quantify this effect, in all of their cases two separate spots with high labeling are present in F5, a ventral one and a more dorsal one. In contrast, $\mathrm{M}$ cells that are predominantly located in the intermediate subregion (bins 3-6) might be more strongly connected to the primary motor area (M1).

\section{Discussion}

We investigated the representation of grip type and object orientation in F5 neurons while macaque monkeys performed a delayed grasping task, in which grip type was instructed by context information (Fig. 1). F5 neurons represented the grip type and object orientation from instruction to movement execution, suggesting that F5 plays an important role for the transformation of object and context information for grasping (Figs. 2, 3; Table 1). Object orientation was represented strongest during instruction, whereas grip-type representation was overall larger and strongest during movement execution (Fig. 4). At the population level, precision grips were overrepresented compared with power grips, and cells preferring middle orientations strongly declined toward movement execution (Fig. 5). Since individual cells kept the same preference for grip type and object orientation across active task epochs (Fig. 6), different subpopulations of cells must be active in different task epochs (Fig. 7). A cell classification based on the tuning onset and offset led to three classes of cells (sensory, sensorimotor, and motor), both for grip type and object orientation (Fig. 8). When comparing both classifications, most orientation-selective cells were also grip-type tuned, whereas motor cells coded predominantly grip type (Table 2 ). Interestingly, the preference ratio for power versus precision grips and for middle versus extreme orientations was markedly different for sensory and latetuned (SM and M) cells (Fig. 9), and motor cells followed a distinct anatomical distribution within F5 (Fig. 10). These results reveal important differences in how grip type and object orientation are processed in F5 and how different cell groups might collaborate to transform sensory signals into grasp movement commands.

\section{Functional connectivity}

The representation of grip type and object orientation in F5 increased rapidly after cue instruction with object orientation leading by $\sim 90 \mathrm{~ms}$. This might indicate different processing pathways for object orientation and grip type instruction.
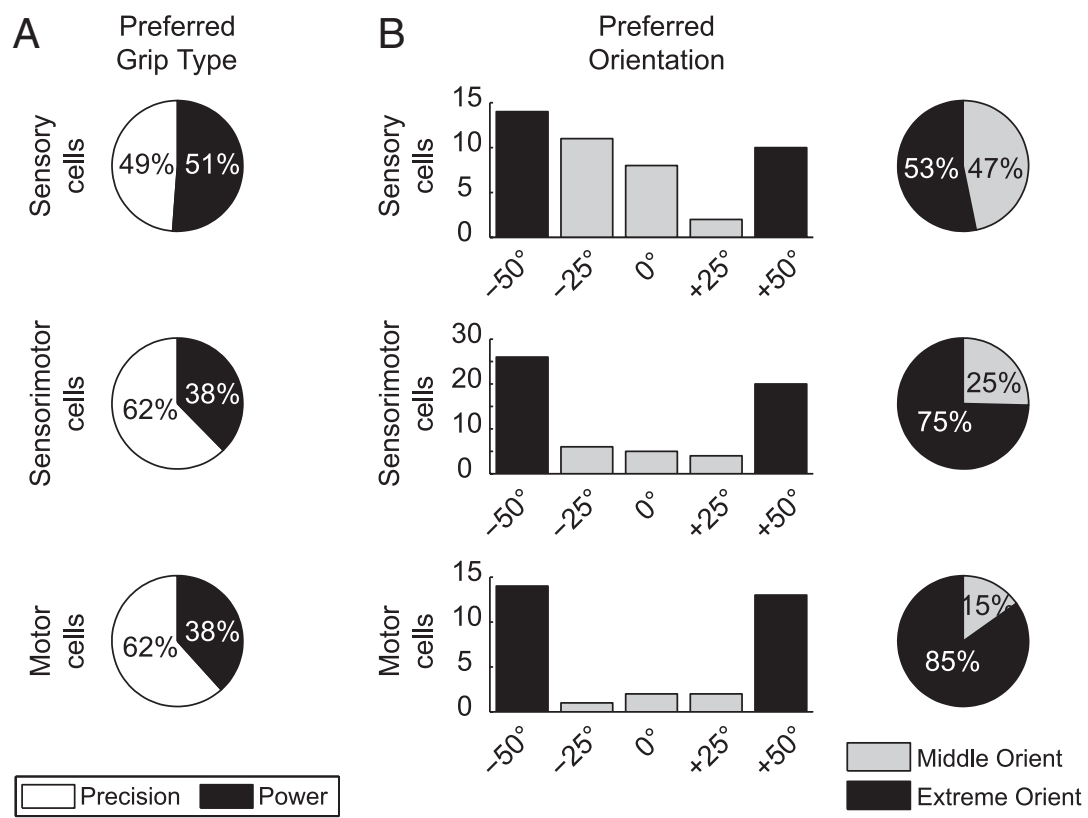

Figure 9. Specific tuning for grip type and orientation (orient) in cell classes. $A$, Percentage of grip type-specific cells preferring precision (white) versus power grip (black) for sensory, sensorimotor, and motor cells. $\boldsymbol{B}$, Histogram, Number of cells preferring individual object orientations $\left(-50^{\circ},-25^{\circ}, 0^{\circ}, 25^{\circ}, 50^{\circ}\right)$ for sensory, sensorimotor, and motor cells. Pie chart, Percentage of orientation-specific cells preferring extreme orientations (black) or middle orientations (gray).
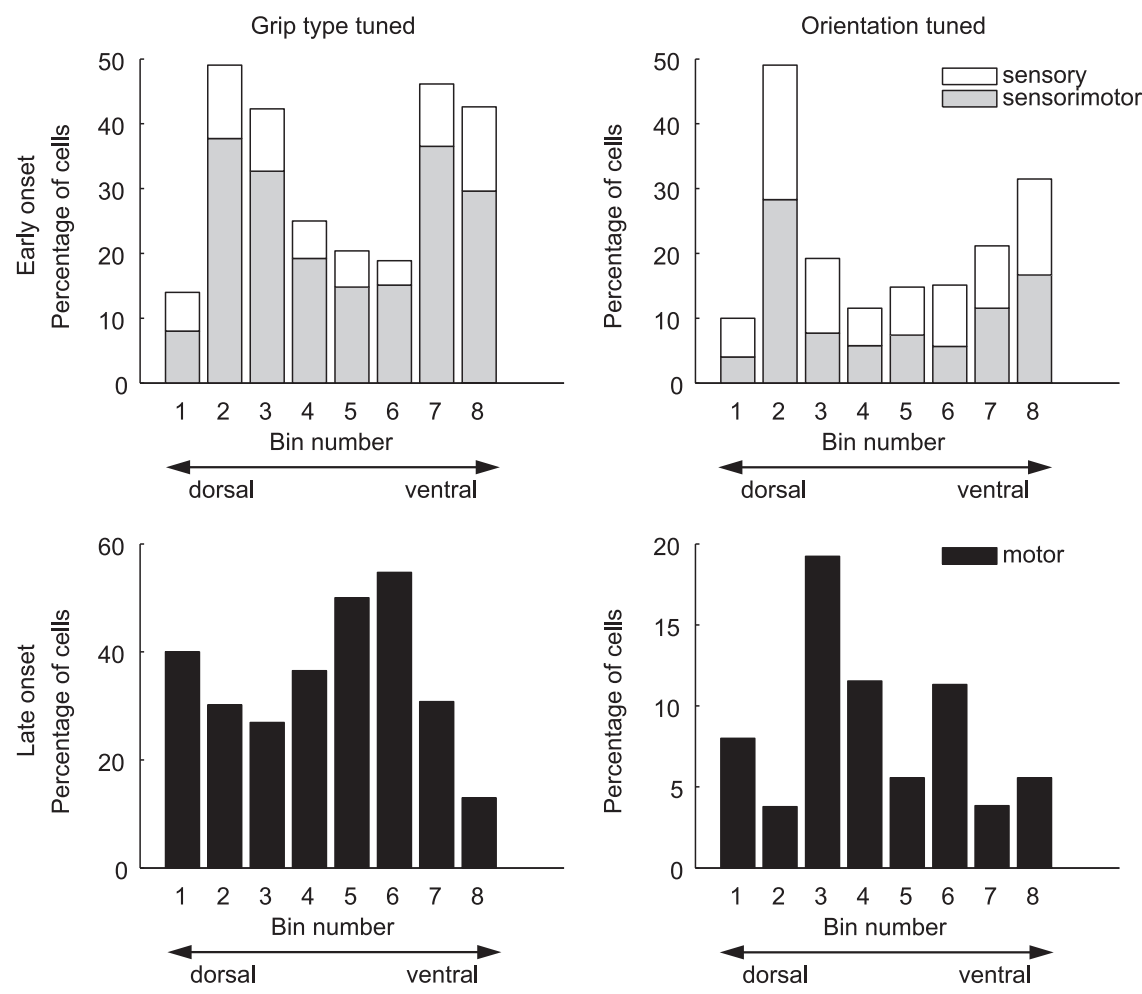

Figure 10. Anatomical distribution of cell classes. Percentage of sensory (white bars), sensorimotor (gray bars), and motor cells (black bars) for grip type (left) and object orientation (right) as a function of their position along the inferior arcuate sulcus.

Object information is most likely propagated from visual and parietal areas to F5. In particular, area AIP contains a strong representation of objects (Baumann et al., 2009) and is strongly and reciprocally connected to F5 (Matelli et al., 1986; TannéGariépy et al., 2002; Borra et al., 2008). The medial posterior parietal area (V6A) also represents object orientation before 
grasping (Fattori et al., 2009) and might potentially also contribute to grasp planning (Battaglini et al., 2002; Fattori et al., 2010).

Grip type, on the other hand, was instructed by a context cue (colored light), which required retrieving a learned association for correct interpretation. Brain areas involved in such arbitrary visuomotor mapping, in addition to the premotor cortex, are the prefrontal cortex, the hippocampus, and the basal ganglia (Murray et al., 2000). F5 receives projections from prefrontal cortex (Matelli et al., 1986; Barbas and Pandya, 1987; Petrides and Pandya, 2002), and this multisynaptic pathway could therefore explain the longer processing time for context information.

Our observation that many F5 cells represent the grip type at the time of movement execution corresponds well with the strong direct connections of F5 to primary motor cortex (Matsumura and Kubota, 1979; Muakkassa and Strick, 1979; Matelli et al., 1986; Dum and Strick, 2005) and the spinal cord (Dum and Strick, 1991; He et al., 1993; Borra et al., 2010). This suggests a fairly direct role of F5 for generating hand-grasping movements.

The weak representation of object orientation during movement could indicate that wrist orientation, necessary to align the hand with the object, is perhaps more strongly represented elsewhere, e.g., in dorsal premotor cortex (Raos et al., 2004; Stark et al., 2007). However, object orientation is clearly present in F5, suggesting that it might be necessary for the selection of grip types or for motor coordination.

Our evidence that cells in F5 are functionally clustered is in contrast to Raos et al. (2006), who studied a fourfold smaller dataset (108 cells). Subregions with predominantly sensory activity (S and SM cells) colocated in two spots most ventrally and dorsally in F5. They seem to coincide with regions of strong connectivity to AIP (Borra et al., 2008, their Fig. 3), whereas the intermediate region of our recordings was predominantly active during movement execution ( $\mathrm{M}$ cells). This intermediate region is likely located well within the posterior subdivision of F5 (F5p) (Nelissen et al., 2005; Belmalih et al., 2009), which is strongly connected to the hand area of primary motor cortex (Matsumura and Kubota, 1979; Muakkassa and Strick, 1979; Matelli et al., 1986; Dum and Strick, 2005). These functional connections place F5 right at the center of cortical hand movement control.

\section{Comparison to previous work}

Previous studies of F5 have classified neurons in F5 as motor or visuomotor depending on the neural activity during grasping in the light and dark (Murata et al., 1997; Raos et al., 2006). Motor neurons were active only during movement execution, whereas visuomotor neurons responded during object presentation. This is compatible with our definition of M and SM cells. In our task, however, visual information was present only during cue and not in the following planning and movement execution epochs. Nonetheless, F5 encoded the instructed grip type during cue and planning in the absence of vision, which highlights its role for sensorimotor transformation.

The decline of orientation tuning during the course of the task was not observed by Raos et al. (2006), which is likely explained by the fact that their objects remained visible throughout the task. This also prevented the detection of sensory cells.

\section{Comparison with AIP}

Our recordings were made in the same animals and often during the same sessions as recordings in AIP with the same paradigm (Baumann et al., 2009). This allowed a direct comparison with results in AIP. As expected from their strong direct and reciprocal connections (Matelli et al., 1986; Luppino et al., 1999; Borra et al.,
2008), grip type and object orientation were represented similarly in F5 and AIP. For example, AIP cells also showed a bimodal distribution of the onset of grip-type tuning with peaks during the cue and movement epoch, and a large percentage of AIP cells represented object orientation throughout the task (Baumann et al., 2009). These findings strongly suggest that sensorimotor transformation for hand grasping occurs in a distributed network including premotor and parietal cortices, similar to what is known for eye and arm movements control (Rizzolatti and Luppino, 2001; Andersen and Buneo, 2002; Gold and Shadlen, 2007; Pesaran et al., 2008; Gail et al., 2009).

The main difference to F5 is that AIP represents object orientation more strongly and persistently. This is evident from the fraction of orientation-tuned cells in the movement epoch (AIP, 55\%; F5, 20\%) and throughout the task (AIP, 77\%; F5, 43\%) (Baumann et al., 2009). Furthermore, the representation of object orientation in F5 is much weaker and more coupled to griptype representation. Nevertheless, object information is present in F5, in particular in S and SM cells, which could simply reflect the strong connectivity to AIP, or might be necessary for appropriate grip-type selection or the coordination of grasping and reaching actions (Jeannerod et al., 1995). In this respect, it remains to be seen whether F5 neurons code the object orientation or the intended wrist orientation of the upcoming movement.

\section{Possible coding schemes}

The preference for a particular grip type or object orientation remained largely constant in individual cells (Fig. 6). Our neural classification based on tuning onset and offset was therefore justified. But which roles do these cell classes play for the sensorimotor processing of hand movements?

$S$ cells preferred power and precision grips in equal numbers and likewise represented object orientations almost equally. This could point to a high-level (visual, cognitive, or abstract) coding scheme that places equal weight on different alternatives. In contrast, SM and M cells showed an overrepresentation of precision grips and extreme orientations. This could indicate a motor code, since precision grips are arguably more difficult to perform and might require more cortical resources than power grips. Likewise, the motor code of wrist orientation might be implemented in a push-pull mechanism for pronation and supination, which could require a strong representation of extreme orientations. Therefore, the coding scheme of SM and M cells could be regarded as more motor-related than abstract, whereas $\mathrm{S}$ cells might encode a high-level representation. In contrast, sensory and sensorimotor cells in AIP have been classified as sensory or abstract (Baumann et al., 2009). In other words, the coding scheme in AIP is determined by the tuning onset, whereas in F5 it is the tuning offset. This places F5 neurons closer to the motor cortex, whereas AIP codes grasp movements predominantly in sensory or abstract terms during movement preparation.

In summary, our results suggest that F5 is involved in contextspecific grasp movement planning, for which different subpopulations are functionally connected to parietal, motor, and frontal areas. This emphasizes an integrative role of F5 for the planning of hand movements on the basis of sensory, contextual, and, most likely, volitional information.

\section{References}

Amemori K, Sawaguchi T (2006) Rule-dependent shifting of sensorimotor representation in the primate prefrontal cortex. Eur J Neurosci 23:1895-1909.

Andersen RA, Buneo CA (2002) Intentional maps in posterior parietal cortex. Annu Rev Neurosci 25:189-220. 
Barbas H, Pandya DN (1987) Architecture and frontal cortical connections of the premotor cortex (area 6) in the rhesus monkey. J Comp Neurol 256:211-228.

Battaglini PP, Muzur A, Galletti C, Skrap M, Brovelli A, Fattori P (2002) Effects of lesions to area V6A in monkeys. Exp Brain Res 144:419-422.

Baumann MA, Fluet MC, Scherberger H (2009) Context-specific grasp movement representation in the macaque anterior intraparietal area. J Neurosci 29:6436-6448.

Belmalih A, Borra E, Contini M, Gerbella M, Rozzi S, Luppino G (2009) Multimodal architectonic subdivision of the rostral part (area F5) of the macaque ventral premotor cortex. J Comp Neurol 512:183-217.

Borra E, Belmalih A, Calzavara R, Gerbella M, Murata A, Rozzi S, Luppino G (2008) Cortical connections of the macaque anterior intraparietal (AIP) area. Cereb Cortex 18:1094-1111.

Borra E, Belmalih A, Gerbella M, Rozzi S, Luppino G (2010) Projections of the hand field of the macaque ventral premotor area F5 to the brainstem and spinal cord. J Comp Neurol 518:2570-2591.

Cerri G, Shimazu H, Maier MA, Lemon RN (2003) Facilitation from ventral premotor cortex of primary motor cortex outputs to macaque hand muscles. J Neurophysiol 90:832-842.

Dum RP, Strick PL (1991) The origin of corticospinal projections from the premotor areas in the frontal lobe. J Neurosci 11:667-689.

Dum RP, Strick PL (2005) Frontal lobe inputs to the digit representations of the motor areas on the lateral surface of the hemisphere. J Neurosci 25:1375-1386.

Fattori P, Breveglieri R, Marzocchi N, Filippini D, Bosco A, Galletti C (2009) Hand orientation during reach-to-grasp movements modulates neuronal activity in the medial posterior parietal area V6A. J Neurosci 29: 1928-1936.

Fattori P, Raos V, Breveglieri R, Bosco A, Marzocchi N, Galletti C (2010) The dorsomedial pathway is not just for reaching: grasping neurons in the medial parieto-occipital cortex of the macaque monkey. J Neurosci 30:342-349.

Fogassi L, Gallese V, Buccino G, Craighero L, Fadiga L, Rizzolatti G (2001) Cortical mechanism for the visual guidance of hand grasping movements in the monkey: a reversible inactivation study. Brain 124:571-586.

Gail A, Andersen RA (2006) Neural dynamics in monkey parietal reach region reflect context-specific sensorimotor transformations. J Neurosci 26:9376-9384.

Gail A, Klaes C, Westendorff S (2009) Implementation of spatial transformation rules for goal-directed reaching via gain modulation in monkey parietal and premotor cortex. J Neurosci 29:9490-9499.

Gentilucci M, Scandolara C, Pigarev IN, Rizzolatti G (1983) Visual responses in the postarcuate cortex (area 6) of the monkey that are independent of eye position. Exp Brain Res 50:464-468.

Gentilucci M, Fogassi L, Luppino G, Matelli M, Camarda R, Rizzolatti G (1988) Functional organization of inferior area 6 in the macaque monkey. I. Somatotopy and the control of proximal movements. Exp Brain Res 71:475-490.

Gold JI, Shadlen MN (2007) The neural basis of decision making. Annu Rev Neurosci 30:535-574.

Gottlieb J, Goldberg ME (1999) Activity of neurons in the lateral intraparietal area of the monkey during an antisaccade task. Nat Neurosci 2:906-912.

He SQ, Dum RP, Strick PL (1993) Topographic organization of corticospinal projections from the frontal lobe: motor areas on the lateral surface of the hemisphere. J Neurosci 13:952-980.

Hoshi E, Shima K, Tanji J (2000) Neuronal activity in the primate prefrontal cortex in the process of motor selection based on two behavioral rules. J Neurophysiol 83:2355-2373.

Jeannerod M, Arbib MA, Rizzolatti G, Sakata H (1995) Grasping objects: the cortical mechanisms of visuomotor transformation. Trends Neurosci 18:314-320.

Kakei S, Hoffman DS, Strick PL (2001) Direction of action is represented in the ventral premotor cortex. Nat Neurosci 4:1020-1025.

Kalaska JF, Cisek P, Gosselin-Kessiby N (2003) Mechanisms of selection and guidance of reaching movements in the parietal lobe. Adv Neurol 93:97-119.

Lemon RN, Kirkwood PA, Maier MA, Nakajima K, Nathan P (2004) Direct and indirect pathways for corticospinal control of upper limb motoneurons in the primate. Prog Brain Res 143:263-279.

Luppino G, Murata A, Govoni P, Matelli M (1999) Largely segregated parietofrontal connections linking rostral intraparietal cortex (areas AIP and VIP) and the ventral premotor cortex (areas F5 and F4). Exp Brain Res 128:181-187.

Matelli M, Camarda R, Glickstein M, Rizzolatti G (1986) Afferent and efferent projections of the inferior area 6 in the macaque monkey. J Comp Neurol 251:281-298.

Matsumura M, Kubota K (1979) Cortical projection to hand-arm motor area from post-arcuate area in macaque monkeys: a histological study of retrograde transport of horseradish peroxidase. Neurosci Lett 11:241246.

Muakkassa KF, Strick PL (1979) Frontal lobe inputs to primate motor cortex: evidence for four somatotopically organized 'premotor' areas. Brain Res 177:176-182.

Muir RB, Lemon RN (1983) Corticospinal neurons with a special role in precision grip. Brain Res 261:312-316.

Murata A, Fadiga L, Fogassi L, Gallese V, Raos V, Rizzolatti G (1997) Object representation in the ventral premotor cortex (area F5) of the monkey. J Neurophysiol 78:2226-2230.

Murata A, Gallese V, Luppino G, Kaseda M, Sakata H (2000) Selectivity for the shape, size, and orientation of objects for grasping in neurons of monkey parietal area AIP. J Neurophysiol 83:2580-2601.

Murray EA, Bussey TJ, Wise SP (2000) Role of prefrontal cortex in a network for arbitrary visuomotor mapping. Exp Brain Res 133:114-129.

National Research Council (2003) Guidelines for the care and use of mammals in neuroscience and behavioral research. Washington, D.C.: National Academies.

Nelissen K, Luppino G, Vanduffel W, Rizzolatti G, Orban GA (2005) Observing others: multiple action representation in the frontal lobe. Science 310:332-336

Pesaran B, Nelson MJ, Andersen RA (2008) Free choice activates a decision circuit between frontal and parietal cortex. Nature 453:406-409.

Petrides M, Pandya DN (2002) Comparative cytoarchitectonic analysis of the human and the macaque ventrolateral prefrontal cortex and corticocortical connection patterns in the monkey. Eur J Neurosci 16:291-310.

Raos V, Umiltá MA, Gallese V, Fogassi L (2004) Functional properties of grasping-related neurons in the dorsal premotor area $\mathrm{f} 2$ of the macaque monkey. J Neurophysiol 92:1990-2002.

Raos V, Umiltá MA, Murata A, Fogassi L, Gallese V (2006) Functional properties of grasping-related neurons in the ventral premotor area F5 of the macaque monkey. J Neurophysiol 95:709-729.

Rizzolatti G, Luppino G (2001) The cortical motor system. Neuron 31:889-901.

Sakata H, Taira M, Murata A, Mine S (1995) Neural mechanisms of visual guidance of hand action in the parietal cortex of the monkey. Cereb Cortex 5:429-438.

Scherberger H, Andersen RA (2007) Target selection signals for arm reaching in the posterior parietal cortex. J Neurosci 27:2001-2012.

Stark E, Asher I, Abeles M (2007) Encoding of reach and grasp by single neurons in premotor cortex is independent of recording site. J Neurophysiol 97:3351-3364.

Taira M, Mine S, Georgopoulos AP, Murata A, Sakata H (1990) Parietal cortex neurons of the monkey related to the visual guidance of hand movement. Exp Brain Res 83:29-36.

Tanné-Gariépy J, Rouiller EM, Boussaoud D (2002) Parietal inputs to dorsal versus ventral premotor areas in the macaque monkey: evidence for largely segregated visuomotor pathways. Exp Brain Res 145:91-103.

Umilta MA, Brochier T, Spinks RL, Lemon RN (2007) Simultaneous recording of macaque premotor and primary motor cortex neuronal populations reveals different functional contributions to visuomotor grasp. J Neurophysiol 98:488-501.

Wallis JD, Anderson KC, Miller EK (2001) Single neurons in prefrontal cortex encode abstract rules. Nature 411:953-956.

White IM, Wise SP (1999) Rule-dependent neuronal activity in the prefrontal cortex. Exp Brain Res 126:315-335.

Zar JH (1999) Biostatistical analysis. Upper Saddle River, New Jersey: Prentice-Hall. 